\title{
EFFECTS OF GALANIN N-TERMINAL FRAGMENT (1-15) IN THE LIGHT/DARK AND TAIL SUSPENSION TESTS.
}

A.Flores-Burgess $^{1,2}$, C.Millón Peñuela ${ }^{1}$, M.A.Narváez Peláez ${ }^{1}$, L.Santín ${ }^{2}$, J.A.Narváez Bueno ${ }^{1}$, Z.DíazCabiale $^{1}$

Galanin N-terminal fragment (1-15) [Gal(1-15)] is involved in mood regulation. The intracerebroventricular (icv) administration of Gal(1-15) produces a depressive-like behaviour in the forced swim test (FST) and an anxiety-like behaviour in the open field test (OF) in rats. In this work we analyze the effect of Gal(1-15) in two more behavioural tests, the tail suspension test (TLT) and the light/dark test.

Gal(1-15) (3nmol), effective dose in FST and OF, or artificial cerebrospinal were injected in animals $(n=5-8) 15$ minutes before the test. Behavioural assessment were conducted with at least one week between tests. Student's $t$-test was used for comparision between experimental groups.

In the light/dark test we analyzed during $5 \mathrm{~min}$ three parametres as indicators of anxiety-like behaviour. Gal(1-15) significantly reduced the time spent in the light compartiment by $52 \%(\mathrm{p}<0.05)$ and the latency time for entering the dark box by $65 \%$ $(p<0.05)$. An increased in the latency time for re-entering the light box was also observed $(\mathrm{p}<0.05)$.

In the TST, total immobility time was analyzed during 6 min test as parameter indicator of depressive-like behaviour. Gal(1-15) significantly increased rat immobility by $16 \%$ $(\mathrm{p}<0.05)$.

Our results describe that Gal(1-15) exerts strong depressive- and anxiety-like effects in these tests, indicating a potential role of Gal(1-15) in mood disorders. These results may give the basis for the development of novel therapeutic drugs targeting Gal(1-15) for depression and anxiety.

This work has been supported by the Junta de Andalucia CVI6476 Spanish Ministry of Economy and Competitiveness (PSI2013-44901-P to L.J.S). 